\title{
Human Umbilical Cord Blood Stem Cells and Chitosan Hydrogels Produce Similar Beneficial Effects in Acute Myocardial Infarctions and Ischemic Cardiomyopathies
}

\author{
Robert J Henning*, Abraham Khan, David Whitaker and Narendra Sastry
}

Departments of Medicine and Cardiothoracic Surgery, James A. Haley Veterans' Hospital, University of South Florida, USA

*Corresponding author: Robert J Henning, MD, Departments of Medicine and Cardiothoracic Surgery, James A. Haley Veterans' Hospital, University of South Florida, Tampa, Florida, USA, E-mail: robert.henning@va.gov

\section{Abstract}

We utilized two separate techniques, human Umbilical Cord Blood Stem Cells (hUCBC) and chitosan hydrogels, to limit acute myocardial infarction size and LV remodeling. Human Umbilical Cord Blood Mononuclear Cells (hUCBC), contain hematopoietic and mesenchymal stem cells. Chitosan is a polysaccharide. Chitosan hydrogels are approved by the United States Food and Drug Administration for human use in drug delivery and are used in repair of skin, nerve, cartilage, and bone. Hydrogels made from chitosan are liquid at room temperature but form a gel matrix at body temperature. We permanently ligated the left anterior descending coronary artery of 100 rats and then randomly divided the rats into control, hUCBC, or chitosan gel treatments. Transthoracic echocardiograms were obtained on rats prior to infarction and then at two, four, and eight weeks after infarction. Hearts were harvested from randomly selected rats from each group at each time for infarct size. Wall thickness and neovascularization of the infarction border zone were determined at eight weeks after infarction. Infarct sizes in the controls, expressed as a percentage of total right plus left ventricular area, averaged $25 \pm 1 \%$ at 2 weeks, 26.5 $\pm 1 \%$ at 4 weeks and $27 \pm 1 \%$ at 8 weeks. Infarct sizes in the hUCBC Group averaged $16 \pm 1 \%$ at two weeks and 14 $\pm 1 \%$ at 4 weeks and at 8 weeks (all $p<0.001$ compared with controls) and in the Gel Group averaged $17 \pm 1 \%$ at two weeks, $14.5 \pm 0.9 \%$ at 4 weeks and $13.9 \pm 0.8 \%$ at 8 weeks (all $p<0.01$ compared with controls). There was no significant difference in infarct size between the hUCBC and the Gel group. Injection of hUCBC or Gel into infarct borders produced scars that were similar in thickness and 4-5 mm thicker than the control scars at 8 weeks $(p<0.01)$. LV fractional shortening decreased in controls from a normal value of $54 \pm 1 \%$ and averaged $24 \pm 1.1 \%$ at 2 weeks. $16.8 \pm 1.2 \%$ at 4 weeks, and $19.9 \pm 1.1 \%$ at 8 weeks (all $p<0.001$ compared with normals). Fractional shortening in hUCBC Group averaged $33.1 \pm 0.9$ at 2 weeks, $33.5 \pm 1.1 \%$ at 4 weeks
\end{abstract}

and $34 \pm 0.9 \%$ at 8 weeks and in the Gel group averaged $31 \pm 1 \%$ at 2 weeks, $32 \pm 1.2 \%$ at 4 weeks and $32 \pm 0.9$ at 8 weeks (all $p<0.001$ compared with controls). The LV End-Diastolic Diameter (LVED) increased in controls from a normal value of $0.61 \pm 0.05 \mathrm{~cm}$ to $0.88 \pm 0.03 \mathrm{~cm}$ at two weeks then $0.89 \pm 0.01 \mathrm{~cm}$ at 4 weeks, and $0.92 \pm 0.05$ $\mathrm{cm}$ at 8 weeks (all $\mathrm{p}<0.001$ compared with normals). In contrast, LV end-diastolic diameters in the hUCBC and Gel Groups were 17 to $23 \%$ smaller between two and eight weeks after myocardial infarction $(p<0.05)$. There was no significant difference in the hemodynamic measurements between the hUCBC and Gel groups. Vessel density in the border zones of myocardial infarctions after 8 weeks were: $5.3 \pm 0.4 / \mathrm{HPF}$ in the hUCBC treated rats and $4.5 \pm 0.5 /$ HPF in the Gel group in comparison with $3.0 \pm 0.3 / \mathrm{HPF}$ in the DMEM treated rats ( $p<0.01$ compared with controls). We conclude that hUCBC and chitosan hydrogels produce similar and significant reductions in infarct size and LV remodeling and substantial increases in LV border zone wall thickness and vascularity.

\section{Keywords}

Myocardial infarction, Ischemic cardiomyopathy, Cardiac repair, Stem cells, Paracrine factors, Chitosan, Hydrogels, Ventricular remodeling, Ventricular wall stress

\section{Introduction}

Five hundred and twenty-five thousand individuals experience a new acute myocardial infarction each year in the United States [1]. Although the hospital mortality from acute myocardial infarction has decreased to approximately $2 \%$ in the United States, the incidence of patients who develop ischemic cardiomyopathy and congestive heart failure after acute myocardial infarction is significantly increasing. Currently, more than

Citation: Henning RJ, Khan A, Whitaker D, Sastry N (2017) Human Umbilical Cord Blood Stem Cells and Chitosan Hydrogels Produce Similar Beneficial Effects in Acute Myocardial Infarctions and Ischemic Cardiomyopathies. Int J Stem Cell Res Ther 3:047. doi.org/10.23937/2469-570X/1410047

Received: April 21, 2017: Accepted: June 23, 2017: Published: June 26, 2017

Copyright: (C) 2017 Henning RJ, et al. This is an open-access article distributed under the terms of the Creative Commons Attribution License, which permits unrestricted use, distribution, and reproduction in any medium, provided the original author and source are credited. 
550,000 individuals are diagnosed and treated each year for ischemic cardiomyopathies and congestive heart failure [1,2]. New treatments are needed to limit the size of myocardial infarctions and prevent the development of left ventricular remodeling, ischemic cardiomyopathy, and congestive heart failure in patients. In order to accomplish this goal, cardiovascular investigators are administering bone marrow stem cells to patients with acute myocardial infarction. However, the results of adult autologous bone marrow mononuclear/ stem cell administration in the Time Trial, the Late Time Trial, and the Swiss Acute Myocardial Infarction Trial have been disappointing with no significant differences reported between treated patients and patients treated with current standard medical therapy [3]. This may be due, in part, to the fact that bone marrow mononuclear cells from patients with advanced age and patients with chronic diseases, such as ischemic heart disease or diabetes mellitus, are often functionally impaired, propagate poorly and have a shortened life span [4,5]. We are investigating human umbilical cord blood stem cells and chitosan hydrogels in the treatment of acute myocardial infarction in order to limit infarction size and limit or prevent left ventricular remodeling and the development of congestive heart failure. Human Umbilical Cord Blood Mononuclear Cells (hUCBC), contain hematopoietic and mesenchymal stem cells. One milliliter of human umbilical cord blood, obtained after full term infant delivery, contains approximately 8000 primitive hematopoietic progenitor cells and 10,000 multipotent stem/progenitor cells [6]. Mesenchymal stem cells are also present in umbilical cord blood in small numbers but are present in larger numbers from Wharton's Jelly in the umbilical cord [7]. Cord blood cells can be injected into the myocardium during cardiac surgery, left heart catheterization, or coronary artery catheterization for the treatment of myocardial infarction and ischemic cardiomyopathies.

Chitosan and chitosan hydrogels are approved by the US Food and Drug Administration for use in tissue engineering, and drug delivery and are currently used in the repair of skin, nerve, cartilage, and bone [8,9]. Hydrogels made from chitosan are liquid at room temperature but undergo gelation and form a matrix at body temperature. Therefore, these gels can be directly injected into the heart during cardiac surgery or injected by catheter into the LV endocardium at cardiac catheterization. Chitosan gels are biocompatible, non-toxic, bacteriostatic, and are porous $[8,9]$. Therefore chitosan gels permit the diffusion of cells and nutrients to the myocardium. In addition, the degree of chitosan deacetylation can be adjusted to optimize the degradation rate of chitosan in the myocardium and other tissues [8]. We are investigating chitosan hydrogel injections into the border zone of myocardial infarctions in order to increase myocardial wall thickness, decrease myocardial wall stress due to the Law of LaPlace, decrease myocardial oxygen requirements, and in this manner limit myocardial infarction size.
The present investigation compares hUCBC effects with chitosan hydrogel effects in limiting acute myocardial infarction size, decreasing left ventricular remodeling, increasing left ventricular wall thickness and stimulating neovascularization in rats.

\section{Materials and Methods}

Male Sprague-Dawley rats, weighing 250-350 g, were housed in a temperature-controlled environment with free access to food and water. On the day of surgery, the rats were anesthetized with Isoflurane $5 \%$ by inhalation, intubated with polyethylene tubing, and mechanically ventilated (Harvard Apparatus) with oxygen and 3 to $5 \%$ Isoflurane. The rats were then placed in the right lateral decubitus position on a heating pad. A thoracotomy was performed in the fourth left intercostal space, the pericardium was opened, and the left anterior descending coronary artery (LAD) was permanently ligated with 5-0 prolene suture $3 \mathrm{~mm}$ below its origin from the aorta. Myocardial infarction in each rat was confirmed after LAD ligation by discoloration and akinesis of the anterior myocardial wall and by ST segment elevation on the electrocardiogram one hour after coronary artery ligation.

Male rats were utilized in the present experiments rather than female rats in order to avoid female hormonal changes that might affect LV remodeling. One hundred male rats were randomly divided into three groups. The control group consisted of rats in which the LAD coronary artery was permanently ligated and, after 1 hour, 0.5 ml Dulbecco's Modified Eagle Medium (DMEM), was injected around the perimeter of each myocardial infarction. The infarct + hUCBC group consisted of rats that underwent LAD ligation and after 1 hour were given $4 \times 10^{6}$ hUCBC in $0.5 \mathrm{ml}$ DMEM around the perimeter of each infarction. The infarct + chitosan group consisted of rats that underwent LAD ligation and after 1 hour were given $0.50 \mathrm{ml}$ chitosan in DMEM around the perimeter of each infarction. The sequence of the experiments was varied.

In each group, the tip of the injection needle for DMEM, hUCBC or Gel was bent 90 degrees in allow the needle to remain parallel with the myocardial wall. Prior to injection, the syringe connected to the needle was aspirated to ensure the needle had not entered the LV cavity. The chest wall of each rat was then closed with 3-0 Vicryl then 3-0 Prolene sutures in three separate layers and the Isoflurane anesthesia was discontinued. The rats were then continuously monitored for approximately one to two hours until alert and ambulatory. Carprofen, $5 \mathrm{mg} / \mathrm{kg}$, SC, was given for analgesia every 12 hours for 48 hours then as needed after surgery. The University of South Florida/James A. Haley Institutional Review and Animal Use Committees approved all of our protocols and procedures. 


\section{Human Umbilical Cord Blood Mononuclear Cells (hUCBC)}

Human umbilical cord blood mononuclear cells or human cord blood were obtained from human cord cell blood banks (CordUse, Saneron Therapeutics, Cambrex) and the specimens did not contain any identifying information of the original donors. The cells were stored at $-196{ }^{\circ} \mathrm{C}$ in liquid nitrogen. The cord blood was rejected if the blood was positive for Human Immunodeficiency Virus (HIV), human T-lymphocytic virus, hepatitis, syphilis or cytomegalovirus. The mononuclear cell fraction of cord blood was obtained by Ficoll density gradient separation and cryopreserved at $-196{ }^{\circ} \mathrm{C}$ in liquid nitrogen. The cryopreserved hUCBC were thawed at $37{ }^{\circ} \mathrm{C}$ and transferred into centrifuge tubes containing Isolyte $\mathrm{S}, \mathrm{pH}$ 7.4 (Braun Medical). The cells were washed three times, centrifuged at $1500 \mathrm{rpm}$ for $7 \mathrm{~min}$, the supernatant discarded and the hUCBC viability determined by Trypan Blue dye exclusion technique. The hUCBC viability was greater than $90 \%$. hUCBC have very large nuclei that occupy approximately $80-85 \%$ of the cell. This morphology readily distinguishes these cells from cardiac myocytes, which have eccentric nuclei in cells with cross striations, and polymorphonuclear leukocytes, which have poly lobed nuclei. The hUCBC were not propagated in culture flasks. Each dose of hUCBC administered was adjusted to deliver a total of $4 \times 10^{6}$ Trypan Blue-negative hUCBC by a precisely calibrated syringe. The hUCBC contained $1-2 \%$ CD34+ cells and approximately $1 \%$ SH2 (CD105) positive cells as determined by fluorescent antibodies to CD34 and SH2 cells that were obtained from Invitrogen and Osiris, respectively, and fluorescent activated cell sorting cytometry (Becton Dickinson) in our facility. A Mac OS9 G-3 computer (Apple Computer) was used for initial cell analysis. Cell Quest (Becton Dickinson), was used for cell acquisition. The data was then analyzed with FlowJo (Tree Star) on a computer. Immune suppression therapy was not given to any rat that received hUCBC. The use of hUCBC in animal studies has been approved by our Institutional Review Board.

\section{Chitosan hydrogel}

Two hundred mg of chitosan (Sigma), which was 75$90 \%$ deacetylated, was dissolved in $10 \mathrm{ml}$ of distilled water to obtain a $2 \%$ chitosan solution and sterilized using liquid autoclaving. Then $1.15 \mathrm{~g}$ of ultrapure $\beta$-Glycerol Phosphate ( $\beta$-GP) (Sigma) was dissolved in $10 \mathrm{ml}$ of distilled water to obtain $11.5 \% \beta-$ GP and the solution was filter sterilized. Eight $\mathrm{ml}$ of chitosan and $2 \mathrm{ml}$ of $\beta$-GP was then mixed with $2.5 \mathrm{ml}$ of $2.5 \%$ sterile hydroxyethyl cellulose in DMEM [10]. The liquid product forms a gel at body temperature $\left(37^{\circ} \mathrm{C}\right)$ in the rat that is gradually degraded over approximately 4 weeks to non-toxic metabolites that are excreted in urine.

\section{Echocardiography}

M-mode and two dimensional transthoracic echo- cardiograms were obtained on rats prior to acute myocardial infarction and then at two, four, and eight weeks after myocardial infarction. This technique is accurate and reproducible in normal rats and rats with myocardial infarction [11]. An echocardiographic system (Siemens/Acuson) with a $7.5 \mathrm{MHz}$ transducer was used. This system has a resolution of $0.5 \mathrm{~mm}$. Rats were very lightly anesthetized with $2 \%$ Isoflurane and then underwent echocardiographic examinations. Two dimensional and M-mode echocardiograms were obtained at the level of the left ventricular papillary muscles and were recorded on the computer hard disc of the echocardiographic system and on compact discs. A minimum of five cardiac cycles were measured and the values averaged for each rat. An investigator and a sonographer, who were unaware of the rats' treatment, analyzed all the echocardiographic images. Linear measurements of the LV were acquired from the parasternal long-axis or apical views. Left ventricular end-systolic diameter was measured after aortic valve closure or measured from the frame in which the left ventricular chamber dimension was the smallest. Left ventricular fractional shortening was determined from LV End-Diastolic diameter (LVED) minus Left Ventricular End-Systolic diameter (LVES) divided by LVED $\times 100$.

\section{Infarct size}

Hearts were harvested from randomly selected anesthetized rats from each group at baseline then at two, four, and eight weeks after myocardial infarction. The permanent suture occlusion of the LAD coronary artery was verified in each heart. The ventricles of each heart were cut into four separate two millimeter slices parallel to the atrioventricular sulcus. Each biventricular slice was rinsed in $0.9 \% \mathrm{NaCL}$ and then was totally immersed and turned every five minutes for forty-five minutes in 1\% Triphenyltetrazolium Chloride (TTC) solution containing $0.2 \mathrm{M}$ Tris at room temperature in a dark room [12]. The myocardial slices were then stored in $10 \%$ formalin for 24 hours. The ventricular slices were then rinsed in saline and photographed with a digital camera. Triphenyltetrazolium forms a red precipitate in the presence of intact dehydrogenase enzymes in normal myocardium whereas infarcted and injured myocardium lacks these enzymes and appears white to light pink in color within 30 minutes after acute coronary occlusion. Tetrazolium does not stain autolytic myocardium and has a diagnostic efficiency of $88 \%$ for infarcted myocardium [12]. Computer imaging software (Image-Pro Plus) was utilized for planimetry of the area of the infarcted myocardium and the area of normal myocardium in the right and left ventricles. The area of infarction and the area of normal right and left ventricles for each slice were summed to determine total infarct area and the total right and left ventricular area for each heart. Infarct size was then expressed as infarct area divided by total left plus right ventricular muscle area. All measurements were done in duplicate and the results were averaged. 


\section{Histopathology}

Two pathologists, who were unaware of the treatment for each rat, examined heart tissue from randomly selected rats from each group. Ventricular tissue in paraffin was deparaffinized and hydrated with water. The tissue was then stained with hematoxylin and eosin, Masson's Trichrome or with Weigert's Iron-Hematoxylin and Safranin-O solution [13]. The slides were examined and the sections of each ventricle photographed with a digital camera.

The vessel density was determined in LV sections stained with antibodies to von Willebrand antigen (Abcam) Ten high magnification microscopic fields spanning the border zone of the infarct of each rat heart were randomly chosen. Vessels with visible lumens and endothelium positive for von Willebrand antigen were counted and expressed as the mean number of vessels/High Power Field (HPF).

\section{Statistical analysis}

All results are expressed as the mean \pm SEM. Differ- ences among groups were tested by Analyses of Variance (ANOVA). The difference between two groups was tested by a Student's t-test. The Bonferroni modification of the t-test was used for repeated statistical analyses for planned comparisons. Tukey's procedure was used for post-hoc comparisons. A value of $p<0.05$ was judged to be significant.

\section{Results}

\section{Infarct size}

Hearts were harvested from randomly selected rats from each of the groups at two, four, and eight weeks after myocardial infarction. The infarct sizes as a percentage of total right plus left ventricular mass in the DMEM treated rats were significantly larger than the infarct sizes in the hUCBC and Gel groups at two, four and eight weeks after permanent LAD coronary artery ligation. The infarct sizes in the control DMEM Group average $25 \pm 1 \%$ at 2 weeks, $26.5 \pm 1 \%$ at 4 weeks and $27 \pm 1 \%$ at 8 weeks. In the hUCBC Group the infarct sizes

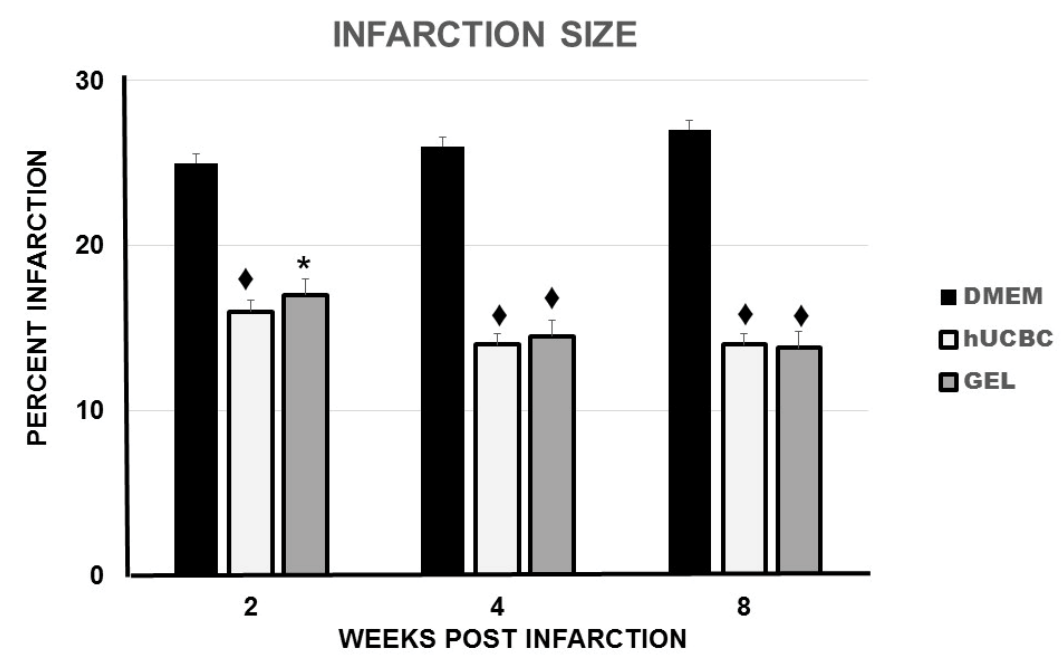

Figure 1: Infarct Size with control DMEM, hUCBC, and Chitosan Hydrogels.

$\checkmark=0.001$ and $^{*}=0.01$ in comparison with DMEM.

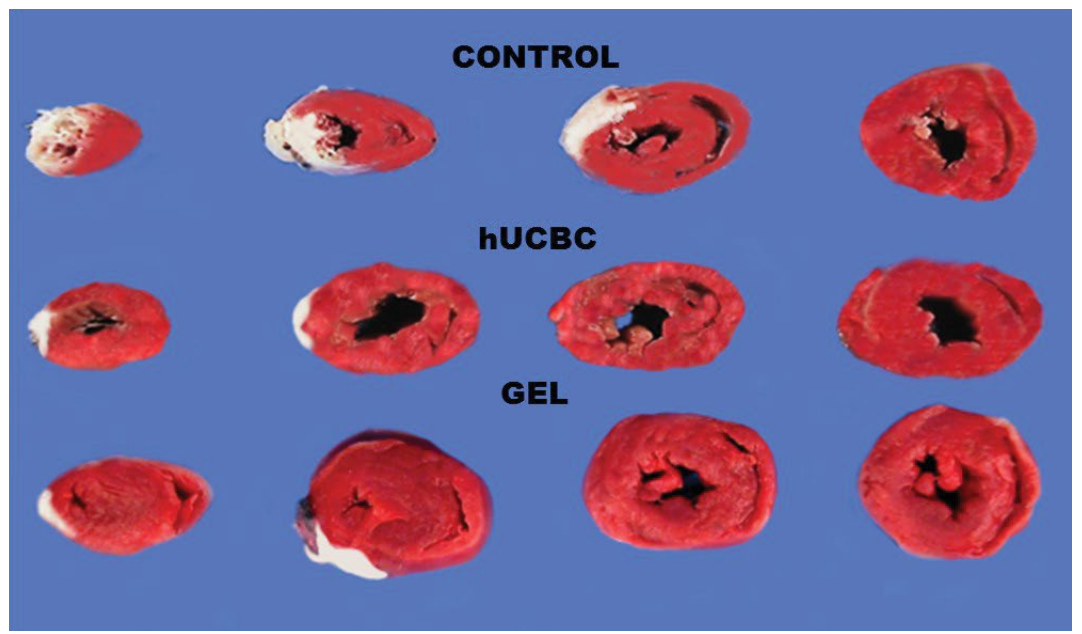

Figure 2: Three representative hearts at eight weeks after myocardial infarction from each of the control DMEM, the hUCBC Group, and the Chitosan Hydrogel Groups. White areas indicate lack of triphenyltetrazolium staining and infarcted myocardium. 
averaged $16 \pm 1 \%$ at two weeks, $14 \pm 1 \%$ at 4 weeks, and $14 \pm 1 \%$ at 8 weeks (all $p<0.001$ in comparison with the DMEM Group). The infarct sizes in the Gel group were similar to and not significantly different from the hUCBC Group and averaged $17 \pm 1 \%$ at two weeks ( $<<0.01$ ), $14.5 \pm 0.9 \%$ at 4 weeks and $13.9 \pm 0.8 \%$ at 8 weeks (both $\mathrm{p}<0.001$ in comparison with the DMEM Group). The decreases in infarct size in the hUCBC Group and in the Gel Group in comparison with the Control Group were most pronounced at two weeks after infarction and treatment (Figure 1). Representative myocardial infarctions from rats from the DMEM Group, hUCBC and Gel Groups are shown in Figure 2. Comparison of the hematoxylin and eosin stained histological sections of the controls, the hUCBC treated hearts, and the Gel treated hearts indicated less myocyte fragmentation and disintegration, less inflammatory mononuclear cell and fibroblast infiltration and less collagen and scar formation in the hUCBC and the chitosan gel treated groups in comparison with the control groups between weeks two and eight post acute infarction. Moreover, we did not detect granuloma formation at any time in any of the three treatment groups. Injection of hUCBC or Gel into the infarct border produced thicker border zone scars at 8 weeks which averaged $0.33 \pm 0.02 \mathrm{~cm}$ in the hUCBC group and $0.32 \pm 0.01 \mathrm{~cm}$ in the Gel group in comparison with the DMEM Group which averaged $0.28 \pm 0.01 \mathrm{~cm}$ (all $\mathrm{p}<0.01$ ). There were few hUCBC identified in the rat myocardium at 8 weeks. Nevertheless, there was no histological or gross anatomical evidence of hUCBC immune rejection in rats that received hUCBC even though immune suppression was not given to any rat.

\section{Left ventricular contractility}

Left ventricular fractional shortening measurements, determined by echocardiography, were used as a measure of LV contractility prior to acute myocardial infarction in all rats and then at two, four, and eight weeks after infarction (Figure 3). Left ventricular fractional shortening averaged $54 \pm 1 \%$ in the rats prior to infarc- tion. In the infarction + control DMEM group, the left ventricular fractional shortening significantly decreased after acute myocardial infarction and averaged $24 \pm$ $1.1 \%$ at 2 weeks. $16.8 \pm 1.2 \%$ at 4 weeks, and $19.9 \pm$ $1.1 \%$ at 8 weeks (all $p<0.001$ in comparison with normal rat LV fractional shortening). The fractional shortening measurements in the hUCBC and Gel treated rats were significantly greater than the measurements in the rats in the DMEM group. The fractional shortening measurements in the hUCBC Group averaged $33.1 \pm 0.9$ at 2 weeks post infarction, $33.5 \pm 1.1 \%$ at 4 weeks, and 34 $\pm 0.9 \%$ at 8 weeks (all $p<0.001$ in comparison with the DMEM group). In the Gel Group the fractional shortening measurements were similar to the hUCBC group and averaged $31 \pm 1 \%$ at 2 weeks, $32 \pm 1.2 \%$ at 4 weeks and $32 \pm 0.9$ at 8 weeks (all $p<0.001$ in comparison with DMEM). The increases in fractional shortening were most pronounced at two weeks after infarction and treatment. There was no significant difference in the fractional shortening measurement between the hUCBC group and the Gel group (Figure 3).

\section{Left ventricular remodeling}

Left Ventricular End-Diastolic (LVED) and Left Ventricular End-Systolic Diameters (LVES) in each rat treatment group after myocardial infarction were used as a measure of left ventricular remodeling. The left ventricular end-diastolic diameters in the normal adult rats averaged $0.61 \pm$ $0.05 \mathrm{~cm}$ prior to myocardial infarction. After infarction, the LVED diameter significantly increased in the control DMEM treated rats to $0.88 \pm 0.03 \mathrm{~cm}$ at two weeks then $0.89 \pm 0.01$ $\mathrm{cm}$ at 4 weeks, and $0.92 \pm 0.05 \mathrm{~cm}$ at 8 weeks all $p<0.001$ in comparison with normal rat LVED (Figure 4A). In contrast, the left ventricular end-diastolic diameters and left ventricular remodeling were significantly less in the hUC$\mathrm{BC}$ and chitosan treated rats. Injection of hUCBC limited LV dilation and remodeling after acute myocardial infarction. In comparison with the rats treated with DMEM, the left ventricular end-diastolic diameters in the hUCBC Group averaged $0.73 \pm 0.04 \mathrm{~cm}$ at 2 weeks $(p<0.05), 0.72 \pm 0.05 \mathrm{~cm}$

FRACTIONAL SHORTENING

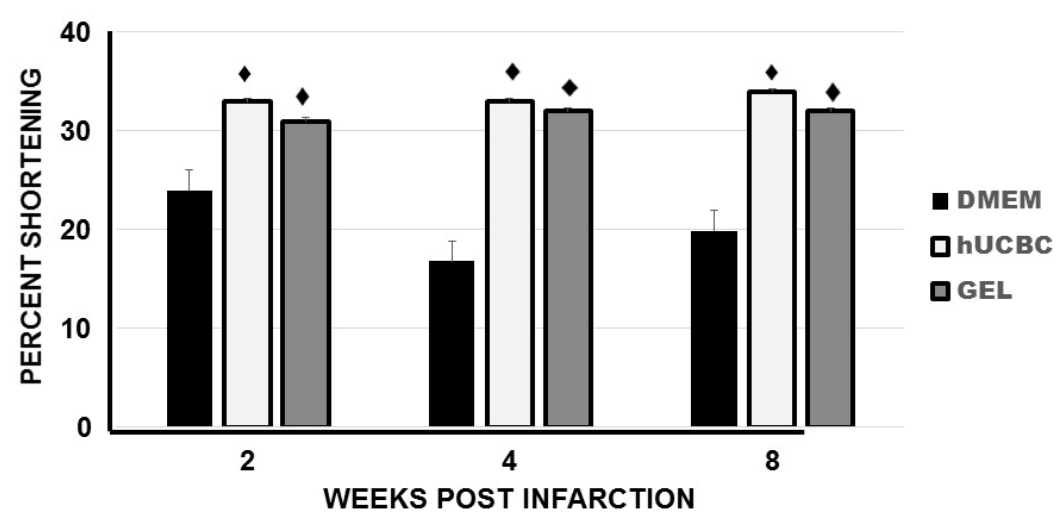

Figure 3: Fractional Shortening. Left ventricular fractional shortening with hUCBC and Chitosan Hydrogels in comparison with rat infarctions treated with DMEM.

$\bullet=0.001$ in comparison with DMEM. 
A

LV END-DIASTOLIC DIAMETER

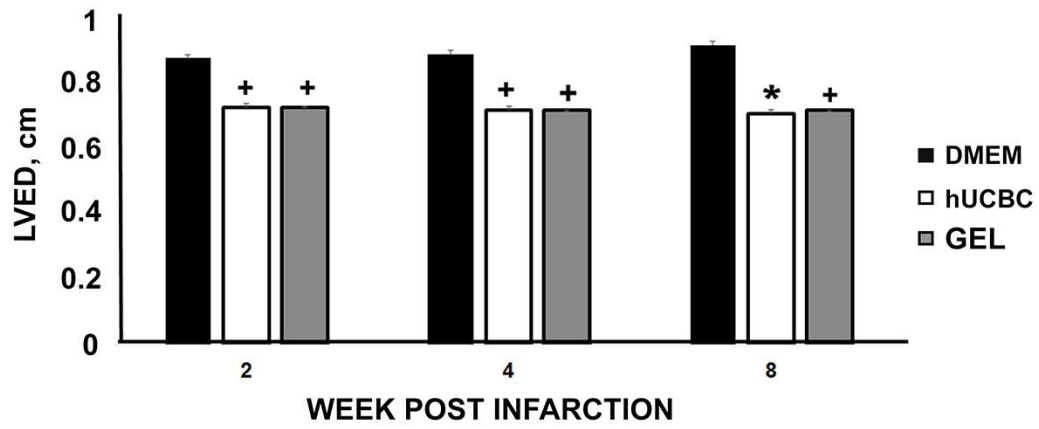

B

LV END-SYSTOLIC DIAMETER

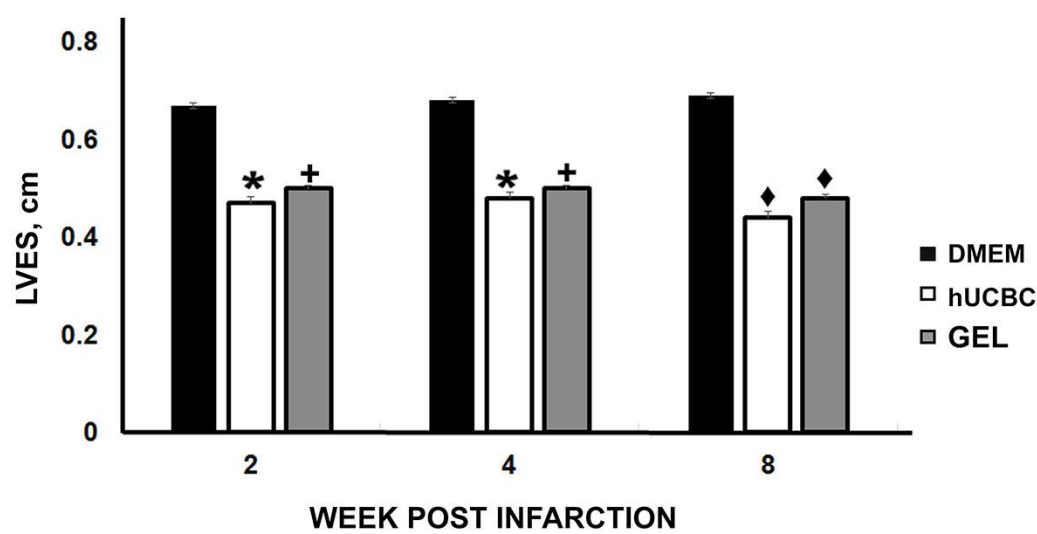

Figure 4A: LV End Diastolic Diameters in rats treated with hUCBC and Chitosan Hydrogel in comparison with rat infarctions treated with DMEM.

${ }^{*}=0.01$ and $^{+}=0.05$ in comparison with DMEM.

Figure 4B: Left Ventricular End Systolic Diameters in rats treated with hUCBC and Chitosan Hydrogels in comparison with rat infarctions treated with DMEM.

$\bullet=0.001,{ }^{*}=0.01$ and $^{+}=0.05$ in comparison with DMEM.

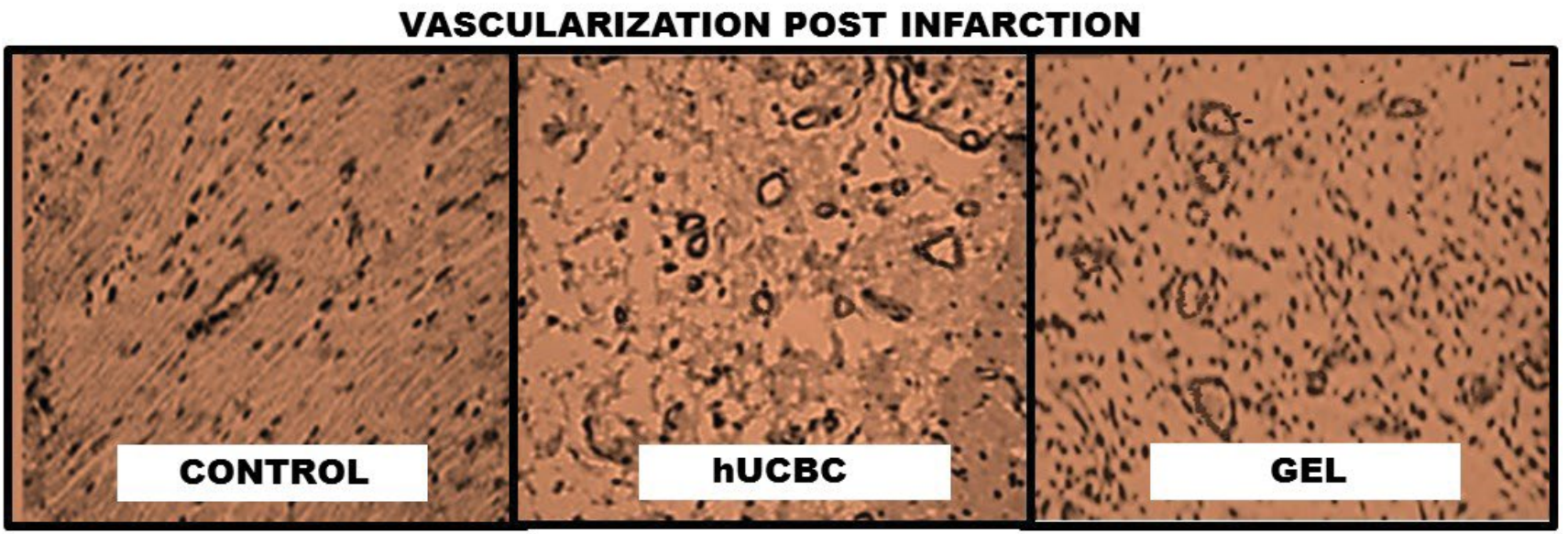

Figure 5: Representative samples of vessel density taken from rat hearts treated with DMEM, hUCBC, or Chitosan Gels. Rat hearts treated with hUCBC or chitosan hydrogels had significantly more blood vessels.

at 4 weeks $(p<0.05)$, and $0.71 \pm 0.03 \mathrm{~cm}$ at 8 weeks $(p<$ $0.01)$. In comparison with rats treated with DMEM, the LV end-diastolic diameters in the chitosan treated rats averaged $0.73 \pm 0.01 \mathrm{~cm}$ at 2 weeks, $0.72 \pm 0.03 \mathrm{~cm}$ at 4 weeks, and $0.72 \pm 0.05 \mathrm{~cm}$ at 8 weeks (all $p<0.05$ ) (Figure $4 \mathrm{~A}$ ).
The decreases in LV remodeling were most pronounced two weeks after infarction and treatment. There was no significant difference between the LV end diastolic diameters between the hUCBC group and the chitosan gel group.

The LV End-Systolic Diameters (LVES) in the rats prior 
to infarction averaged $0.35 \pm 0.05 \mathrm{~cm}$. Permanent occlusion of the left anterior coronary artery significantly increased the LVES at 2 weeks in the DMEM treated rats to $0.67 \pm 0.03 \mathrm{~cm}(p<0.05)$ (Figure 4B). Thereafter the end-systolic diameters in the DMEM Group averaged $0.68 \pm 0.02 \mathrm{~cm}$ at 4 weeks, and $0.69 \pm 0.05 \mathrm{~cm}$ at 8 weeks (all $p<0.001$ in comparison with the normal rat LVES). In contrast, injection of hUCBC prevented the deterioration of LV systolic function in comparison with the DMEM Group at each time. The LV end-systolic diameters in the hUCBC averaged $0.47 \pm 0.05 \mathrm{~cm}$ at 2 weeks ( $p$ $<0.01), 0.48 \pm 0.05 \mathrm{~cm}$ at 4 weeks $(p<0.01), 0.44 \pm 0.01$ $\mathrm{cm}$ at 8 weeks $(\mathrm{p}<0.001)$ (Figure 4B).

Injection of chitosan hydrogel also limited increases in the LV end-systolic diameters and limited the LV remodeling. In comparison with the DMEM treated rats at each time, the LVES in the chitosan treated rats averaged $0.50 \pm 0.04 \mathrm{~cm}$ at 2 weeks $(p<0.05), 0.50 \pm 0.03$ $\mathrm{cm}$ at 4 weeks $(p<0.05)$, and $0.48 \pm 0.04 \mathrm{~cm}$ at 8 weeks ( $p<0.01)$ (Figure 4B). The left ventricular end-systolic diameters were slightly but not significantly larger in the chitosan group in comparison with the hUCBC group.

\section{Angiogenesis}

Vessel density in the border zones of myocardial infarction after 8 weeks of injection were: $5.3 \pm 0.4 / \mathrm{HPF}$ in the hUCBC treated rats and $4.5 \pm 0.5 / \mathrm{HPF}$ in the Gel group in comparison with $3.0 \pm 0.3 / \mathrm{HPF}$ in the DMEM treated rats ( $p<0.01$ compared with controls) (Figure 5). The vessel density was slightly but not significantly greater in the hUCBC group in comparison with the chitosan gel group.

\section{Discussion}

The present investigation demonstrates that hUC$\mathrm{BC}$ and chitosan hydrogels can significantly limit acute myocardial infarction size and left ventricular remodeling and increase the thickness and the vascularity of the infarct border zone. Each of these treatments produces similar and significant improvements in the function of the infarcted heart and decreases in LV remodeling. hUCBC and chitosan hydrogels potentially have direct applicability to the treatment of patients with myocardial infarctions and ischemic cardiomyopathies.

\section{Human umbilical cord blood stem cells cord blood transplantation}

Each year in the United States there are approximately four million live births [14]. Moreover, human cord blood obtained after infant delivery can be frozen for more than 20 years with the recovery of 80 to $90 \%$ of primitive but viable nucleated blood cells [15]. Consequently, cord blood is readily available. The content of hematopoietic progenitor cells in umbilical cord blood varies with gestational age. At 17 weeks gestation, CD34+ hematopoietic cells comprise approximately $11 \%$ of the small mononuclear blood cells in umbilical cord blood while at 38 weeks gestation the CD34+ content is $1-2 \%$ [16]. Approximately 400,000 cord blood units have been stored in blood banks and more than 20,000 allogeneic cord blood cell transplants have been performed worldwide for the treatment of patients with Fanconi anemia, aplastic anemia, beta-thalassemia, severe combined immune deficiency, $\mathrm{X}$-linked lymphoproliferative syndrome, Hurler syndrome, Hunter's syndrome, Wiskott-Aldrich syndrome, acute lymphoid leukemia, acute and chronic myeloid leukemia, myelodysplastic syndrome, and neuroblastoma [17]. In addition, cord blood hematopoietic and mesenchymal stem cells are able to survive during severe hypoxia and maintain their potency due to peroxidases and catalases within these cells, which are able to catalyze free oxygen radicals and thereby significantly limit or prevent umbilical cell necrosis [18-20]. The fact that hUCBC are beneficial in the treatment of different human metabolic and hematological diseases and that cord blood cells can survive during severe ischemia suggests to us that hUCBC can also be beneficial in limiting LV damage from myocardial infarctions.

\section{hUCBC mechanisms of action}

One mechanism whereby hUCBC can be beneficial in ischemic hearts is by stimulating myocardial vasculogenesis and angiogenesis. Vascular endothelial stem cells are normal components of umbilical cord blood that can promote de-novo blood vessel formation and also can integrate into the walls of blood vessels in the periphery of injured tissues and can increase capillary density in ischemic muscle [21-23]. Vascular endothelial stem cells can also release proangiogenic growth factors such as vascular endothelial growth factor, angiopoietin and angiogenin [20,24].

The beneficial effects of hUCBC in ischemic and infarcted myocardium can also be due to their release of growth factors, cytokines, nitric oxide, and inhibitors of metalloproteinases that can limit myocardial inflammation, necrosis and LV remodeling. In this regard, we have demonstrated that hUCBC during severe hypoxia secrete hepatocyte growth factor, anti-inflammatory Interleukin (IL)-10, insulin growth factor, placental growth factor, stem cell factor, and tissue inhibitors of metalloproteinases [24]. Moreover, this paracrine action of hUCBC is increased 50 to $100 \%$ during severe hypoxia and in the presence of free oxygen radicals [20,24]. This paracrine action of hUCBC can suppress in the ischemic myocardium the inflammatory cytokines tumor necrosis factor-alpha, monocyte chemoattraction protein and macrophage inflammatory protein by more than $50 \%$ [25]. Cord blood stem cells can also limit or inhibit caspase expression and the caspase cascade in the myocardium that otherwise contributes to myocardial inflammation and myocyte death after coronary artery occlusion [26]. In addition, hUCBC limit the infiltration and proliferation in acute myocardial infarctions of neu- 
trophils by $75 \%$ and lymphocytes by more than $50 \%$ that otherwise release inflammatory cytokines that cause myocardial inflammation and necrosis $[25,27]$.

Human cord blood stem cell growth factors can activate in ischemic cardiac myocytes and vascular endothelial cells the survival proteins Akt and $\mathrm{Bcl} 2$ by more than $25 \%$ and suppresses the death promoting proteins JNK, p38 and Bad by as much as 30\% [20,24,26]. Activated Akt phosphorylates the cytotoxic protein Bad in myocytes and vascular endothelial cells [24]. The phosphorylated Bad then dissociates from the prosurvival protein $\mathrm{Bcl}-2$, and $\mathrm{Bcl}-2$ limits or prevents the release of cytotoxic mitochondrial proteins into the cytoplasm of myocytes. In this manner, hUCBC can inhibit myocyte death during severe hypoxia, such as occurs with acute myocardial infarction, by more than $40 \%$ [24].

In the present investigation, hUCBC decreased myocardial infarct size and LV remodeling and increased LV fractional shortening and infarct border zone vascularization most likely due to the significant paracrine secretion of growth factors, anti-inflammatory cytokines, and tissue inhibitors of metalloproteinase.

\section{hUCBC immune privilege}

Human umbilical cord blood mononuclear cells contain lymphocytes that are immature and produce significantly smaller amounts of cytokines and immunoglobulins than adult lymphocytes $[27,28]$. Consequently, little or no cytotoxic activity is generated with cord blood cells after rat or human allogeneic cell stimulation $[27,28]$. Moreover, few hUCBC express class II HLA antigens [27]. The immaturity of cord blood lymphocytes and the lack of hUCBC class II HLA antigens explain in the present investigation the absence of immunological reactions in the rats treated with hUCBC during the 8 weeks of observation even though immune suppression therapy was not given to any rat.

\section{Chitosan hydrogels}

Chitosan hydrogel structure: Chitosan is a linear polysaccharide, consisting of $\mathrm{D}$-glucosamine and $\mathrm{N}$-acetyl-D-glucosamine linked by glycosidic bonds. Chitosan hydrogels consist of a mixture of chitosan, $\beta$-glycerol phosphate, and hydroxyethyl cellulose $[29,30]$. The degree of chitosan deacetylation determines the rate of biodegradation in tissue and can be adjusted to optimize the degradation rate of chitosan in the myocardium [29,31]. Chitosan that is 75-90\% deacetylated, which was used in the present investigation, is biodegradable but chitosan that is $>95 \%$ deacetylated is significantly more resistant to degradation in the LV myocardium [29]. This ability to vary the degradation rates of chitosan significantly contrasts with other biomaterials, which have been injected into the myocardium such as fibrin glues, which have a short degradation time. Chitosan gels are non-toxic, biocompatible, bioadhesive and antimicrobial in tissue [32]. The hydrogels are porous and therefore permit the diffusion of oxygen and nutrients. Chitosan hydrogels can create a 3-dimensional scaffold that resembles the natural extracellular matrix of the myocardium without distorting the myocardium. The hydrogels are broken down by lysozymes and $\mathrm{N}$-acetyl-beta-D-glucosaminidases into biocompatible chitosan oligosaccharides which are non-toxic metabolites that are excreted in the urine [29,31]. Chitosan and chitosan hydrogels are approved by the US Food and Drug Administration for use in tissue engineering, and drug delivery and are currently used in the repair of skin, nerve, cartilage, and bone [33].

Chitosan mechanisms of action: The beneficial effects of chitosan in the treatment of acute myocardial infarction in the present investigation can be due to three mechanisms: 1) An increase in LV myocardial stiffness that decreases myocardial wall stress and myocardial oxygen requirements; 2) An acceleration of infarct wound healing; and 3) Stimulation of myocardial angiogenesis [34]. Chitosan hydrogels can provide structural support to the infarcted myocardial wall which is directly dependent on the location of the gel in the myocardium, the volume of gel added to the myocardium, and the relative stiffness of chitosan in the myocardium. Injection of a gel volume of $0.5-1.5 \mathrm{ml}$ into the perimeter of a myocardial infarction, similar to what was done in the present investigation, can increase LV myocardial stiffness by $20 \%$ [35]. This gel volume, which increases LV wall thickness, can reduce the myocardial fiber stresses in the border zone of the infarction by 17 to $20 \%$ from mean LV end-systolic levels of $212 \mathrm{mmHg}$ to near normal levels of $175 \mathrm{mmHg}$ due to the Law of LaPlace [34,35]. This wall stress of $175 \mathrm{mmHg}$ in the border zone is similar to the wall stress in the remote non-infarcted regions of the myocardium. The reduction in LV wall stress is associated with a decrease in myocardial wall oxygen requirements and therefore a decrease in LV myocyte necrosis. In the present investigation, a decrease in LV myocardial oxygen requirements and a reduction in myocardial necrosis contributed to the decrease in infarct size and LV remodeling and the increase in LV ejection fraction in the gel treated rats in comparison with rats with acute myocardial infarction who received DMEM.

Chitosan hydrogels, especially in the presence of serum, can accelerate wound healing by stimulating the migration and proliferation of fibroblasts into the wound and the production of collagen by fibroblasts $[36,37]$. In addition, chitosan hydrogel cross linkable glycosidic bonds can contribute to wound contracture. By stimulating fibroblast migration into infarctions and increasing collagen production, chitosan can increase infarct scar thickness which we observed in the present investigation. Increased scar thickness, especially in the infarct perimeter, can also limit the seepage of pro-in- 
flammatory and death promoting cytokines from necrotic myocytes into the surrounding non-injured myocardium that might otherwise cause additional myocyte necrosis and LV remodeling.

Several mechanisms can account for the angiogenesis in chitosan treated myocardial infarctions. Chitosan can bind plasma proteins and platelet surface proteins, enhance the release of growth factors from platelets and stimulate fibroblasts that secrete Interleukin-8 (IL-8) [38,39]. Interleukin- 8 can chemoattract vascular endothelial cells into the infarction for angiogenesis [38-40]. Chitosan can also form complexes in infarctions with hyaluronates and heparin sulfates, which in turn bind the growth factors stromal cell-derived factor-1, fibroblast growth factors, and platelet-derived growth factors [39]. Hyaluronic acid binds hematopoietic/endothelial progenitor cells that can contribute to angiogenesis. Heparin sulfates bind stromal-cell-derived factor-1 that chemoattracts hematopoietic cells into infarctions which stimulate capillary formation. Fibroblast growth factors can facilitate in the chitosan porous structure the retention and protection of endothelial cells and endothelial progenitor cells from inflammation and necrosis during infarction which facilitates angiogenesis [41-43]. In addition, chitosan can release glucosamine and $\mathrm{N}$-Acetylglucosamine oligomers and monomers that can stimulate endothelial cell recruitment for angiogenesis. In confirmation of the present findings, other investigators have reported in rats with acute myocardial infarctions that chitosan can stimulate angiogenesis and increase LV fractional shortening that is similar to improvements seen with mesenchymal stem cell treatment [44].

\section{Conclusion}

In summary, in the present investigation hUCBC and chitosan hydrogels produced similar and significant reductions in acute myocardial infarction size and LV remodeling and substantial increases in LV border zone wall thickness and vascularity by different mechanisms. hUCBC and chitosan hydrogels can potentially make important contributions to the treatment of patients with acute myocardial infarctions and ischemic cardiomyopathies. Studies are in progress in our laboratory with hUC$\mathrm{BC}$ and chitosan hydrogels to develop an optimal treatment for patients with acute myocardial infarctions and ischemic cardiomyopathies.

\section{Compliance with Ethical Standards}

\section{Conflict of interest}

This study was funded in part by a grant from the Children's Cardiomyopathy Foundation (2017CCF) to $\mathrm{RJH}$. The remaining authors have no financial or personal relationships with other people or organizations that could potentially and inappropriately influence their work and conclusions. All studies were approved by the Institutional Review Boards of the James A. Haley Hospital/University of South Florida College of Medicine. All applicable international, national, and institutional guidelines for the care and use of laboratory animals were followed and approved by the appropriate institutional committees.

\section{Acknowledgements}

This work was supported, in part, by a grant from the Children's Cardiomyopathy Foundation. RJ Henning participated in the experiments and the manuscript construction, Abraham Khan participated in the experiments, David Whitaker and Narendra Sastry participated in manuscript construction. The authors have no financial or personal relationships with other people or organizations that could potentially and inappropriately influence their work and conclusions.

\section{References}

1. Mozaffarian D, Benjamin EJ, Go AS, Arnett DK, Blaha MJ, et al. (2016) Heart Disease and Stroke Statistics-2016 Update: A Report From the American Heart Association. Circulation 133: e38-e360.

2. Roger VL (2013) Epidemiology of Heart Failure. Circ Res 113: 646-659.

3. Henning RJ (2013) Stem cells for cardiac repair: problems and possibilities. Future Cardiol 9: 875-884.

4. Kissel CK, Lehmann R, Assmus B, Aicher A, Honold J, et al. (2007) Selective functional exhaustion of hematopoietic progenitor cells in the bone marrow of patients with postinfarction heart failure. J Am Coll Cardiol 49: 2341-2349.

5. Fadini GP, Boscaro E, de Kreutzenberg S, Agostini C, Seeger F, et al. (2010) Time course and mechanisms of circulating progenitor cell reduction in the natural history of Type 2 diabetes. Diabetes Care 33: 1097-1102.

6. Hal E Broxmeyer (2010) Cord blood hematopoietic stem cell transplantation. In: Hal E Broxmeyer, StemBook. Cambridge (MA), Harvard Stem Cell Institute, USA.

7. Margossian T, Reppel L, Makdissy N, Stoltz JF, Bensoussan D, et al. (2012) Mesenchymal stem cells derived from Wharton's jelly: comparative phenotype analysis between tissue and in vitro expansion. Biomed Mater Eng 22: 243-254.

8. Jiang T, Kumbar SG, Nair LS, Laurencin CT (2008) Biologically active chitosan systems for tissue engineering and regenerative medicine. Curr Top Med Chem 8: 354-364.

9. Rodríguez-Vázquez $M$, Vega-Ruiz $B$, Ramos-Zúñiga $R$, Saldaña-Koppel DA, Quiñones-Olvera LF (2015) Chitosan and Its Potential Use as a Scaffold for Tissue Engineering in Regenerative Medicine. Biomed Res Int 2015: 821279.

10. Hasan A, Khattab A, Islam MA, Hweij KA, Zeitouny J, et al. (2015) Injectable Hydrogels for Cardiac Tissue Repair after Myocardial Infarction. Adv Sci (Weinh) 2: 1500122.

11. Morgan EE, Faullx MD, McElfresh TA, Kung TA, Zawaneh MS, et al. (2004) Validation of echocardiographic methods for assessing left ventricular dysfunction in rats with myocardial infarction. Am J Physiol Heart Circ Physiol 287: 2049-2053.

12. Adegboyega PA, Adesokan A, Haque AK, Boor PJ (1997) Sensitivity and specificity of triphenyl tetrazolium chloride in the gross diagnosis of acute myocardial infarcts. Arch Pathol Lab Med 121: 1063-1068.

13. Rossomacha E, Hoemann C, Shive M (2004) Simple methods for staining chitosan in biotechnological applications. $J$ Histotechnol 27: 31-36. 
14. Hamilton B, Martin JA, Osterman MJ, Curtin SC, Mathews TJ (2015) Births: Final Data for 2014. Natl Vital Stat Rep 64: $1-64$

15. Broxmeyer HE, Lee MR, Hangoc G, Cooper S, Prasain N, et al. (2011) Hematopoietic stem/progenitor cells, generation of induced pluripotent stem cells, and isolation of endothelial progenitors from 21- to 23.5-year cryopreserved cord blood. Blood 117: 4773-4777.

16. Wyrsch A, dalle Carbonare V, Jansen W, Chklovskaia E, Nissen C, et al. (1999) Umbilical cord blood from preterm human fetuses is rich in committed and primitive hematopoietic progenitors with high proliferative and self-renewal capacity. Exp Hematol 27: 1338-1345.

17. Gluckman E (2009) History of cord blood transplantation. Bone Marrow Transplant 44: 621-626.

18. Kuboyama A, Tanaka S, Kawai K, Kasai H, Morii H, et al. (2011) 8-Hydroxyguanine levels and repair capacity during mouse embryonic stem cell differentiation. Free Radic Res 45: 527-533.

19. Zhang J, Chen GH, Wang YW, Zhao J, Duan HF, et al. (2012) Hydrogen peroxide preconditioning enhances the therapeutic efficacy of Wharton's Jelly mesenchymal stem cells after myocardial infarction. Chin Med J 125: 34723478.

20. Henning RJ, Sanberg P, Jimenez E (2014) Human cord blood stem cell paracrine factors activate the survival protein kinase Akt and inhibit death protein kinases JNK and p38 in injured cardiomyocytes. Cytotherapy 16: 1158-1168.

21. Murohora T, Ikeda H, Duan J, Shintani S, Sasaki Ki, et al. (2000) Transplanted cord blood-derived endothelial precursor cells augment postnatal neovascularization. J Clin Invest 105: 1527-1536.

22. Pesce M, Orlandi A, lachininoto MG, Straino S, Torella AR, et al. (2003) Myoendothelial differentiation of human umbilical cord blood-derived stem cells in ischemic limb tissues. Circ Res 93: e51-e62.

23. Hirata $Y$, Sata M, Motomura N, Takanashi M, Suematsu $Y$, et al. (2005) Human umbilical cord blood cells improve cardiac function after myocardial infarction. Biochem Biophys Res Commun 327: 609-614.

24. Jin H, Sanberg PR, Henning RJ (2013) Human umbilical cord blood mononuclear cell-conditioned media inhibits hypoxic-induced apoptosis in human coronary artery endothelial cells and cardiac myocytes by activation of the survival protein Akt. Cell Transplant 22: 1637-1650.

25. Henning RJ, Shariff M, Eadula U, Alvarado F, Vasko M, et al. (2008) Human cord blood mononuclear cells decrease cytokines and inflammatory cells in acute myocardial infarction. Stem Cells Dev 17: 1207-1219.

26. Henning RJ, Dennis S, Sawmiller D, Hunter L, Sanberg P et al. (2012) Human umbilical cord blood mononuclear cells activate the survival protein Akt in cardiac myocytes and endothelial cells that limits apoptosis and necrosis during hypoxia. Transl Res 159: 497-506.

27. Wang M, Yang Y, Yang D, Luo F, Liang W, et al. (2009) The immunomodulatory activity of human umbilical cord blood derived mesenchymal stem cells in vitro. Immunology 126 : 220-232.

28. Zhao Y, Huang Z, Qi M, Lazzarini P, Mazzone T (2007) Immune regulation of $T$ lymphocyte by a newly characterized human umbilical cord blood stem cell. Immunol Lett 108 : 78-87.
29. Dong W, Han B, Feng Y, Song F, Chang J, et al. (2010) Pharmacokinetics and biodegradation mechanisms of a versatile carboxymethyl derivative of chitosan: in vivo and in vitro evaluation. Biomacromolecules 11: 1527-1533.

30. Jang T, Kumbar SG, Nair L, Laurencin C (2006) Biologically active chitosan systems for tissue engineering and regenerative medicine. Current Topics in Medicinal Chem 8: 354364.

31. Chenite A, Chaput C, Wang D, Combes C, Leroux J, et al. (2000) Novel injectable neutral solutions of chitosan form biodegradable gels in situ. Biomaterials 21: 2155-2161.

32. Azuma K, Izumi R, Osaki T, Ifuku S, Morimoto $M$, et al. (2015) Chitin, chitosan, and its derivatives for wound healing: old and new materials. J Funct Biomater 6: 104-142.

33. Bergera J, Reista M, Mayer JM, Felt O, Peppas NA, et al. (2004) Structure and interactions in covalently and ionically crosslinked chitosan hydrogels for biomedical applications. Eur J Pharm Biopharm 57: 19-34.

34. Henning RJ, Khan A, Jimenez E (2016) Chitosan hydrogels significantly limit left ventricular infarction and remodeling and preserve myocardial contractility. J Surg Res 201: 490497.

35. Wall ST, Walker JC, Healy KE, Ratcliffe MB, Guccione JM (2006) Theoretical impact of the injection of material into the myocardium: a finite element model simulation. Circulation 114: $2627-2635$

36. Howling GI, Dettmar PW, Goddard PA, Hampson FC, Dornish M, et al. (2001) The effect of chitin and chitosan on the proliferation of human skin fibroblasts and keratinocytes in vitro. Biomaterials 22: 2959-2966.

37. Uslu B, Biltekin B, Denir S, Ozbas-Turan S, Arbak S, et al. (2015) Differences between solution and membrane forms of chitosan on the in vitro activity of fibroblasts. Balkan Med J 32: 69-78.

38. Ishihara $\mathrm{M}$, Ono $\mathrm{K}$, Sato $\mathrm{M}$, Nakanishi $\mathrm{K}$, Saito $\mathrm{Y}$, et al. (2001) Acceleration of wound contraction and healing with a photocrosslinkable chitosan hydrogel. Wound Repair Regen 9: 513-521.

39. Mori T, Okumura M, Matsuura M, Ueno K, Tokura S, et al. (1997) Effects of chitin and its derivatives on the proliferation and cytokine production of fibroblasts in vitro. Biomaterials 18: 947-951.

40. Fischer TH, Thatte HS, Nichols TC, Bender-Neal DE, Bellinger $A D$, et al. (2005) Synergistic platelet integrin signaling and factor XII activation in poly-N-acetyl glucosamine fiber-mediated hemostasis. Biomaterials 26: 5433-5443.

41. Deng C, Zhang P, Vulesevic B, Kuraitis D, Li F, et al. (2010) A collagen-chitosan hydrogel for endothelial differentiation and angiogenesis. Tissue Eng Part A 16: 3099-3109.

42. Madihally SV, Matthew HW (1999) Porous chitosan scaffolds for tissue engineering. Biomaterials 20: 1133-1142.

43. Chupa JM, Foster AM, Sumner SR, Madihally SV, Matthew HW (2000) Vascular cell responses to polysaccharide materials: in vitro and in vivo evaluations. Biomaterials 21 : 2315-2322.

44. Xu B, Li Y, Deng B, Liu X, Wang L, et al. (2017) Chitosan hydrogel improves mesenchymal stem cell transplant survival and cardiac function following myocardial infarction in rats. Exp Ther Med 13: 588-594. 